\title{
The power graph of a torsion-free group
}

\author{
Peter J. Cameron $^{1}{ }_{D}$ • Horacio Guerra ${ }^{1,2}$ • Šimon Jurina ${ }^{1}$
}

Received: 1 July 2017 / Accepted: 19 February 2018 / Published online: 28 February 2018

C Springer Science+Business Media, LLC, part of Springer Nature 2018

\begin{abstract}
The power graph $P(G)$ of a group $G$ is the graph whose vertex set is $G$, with $x$ and $y$ joined if one is a power of the other; the directed power graph $\vec{P}(G)$ has the same vertex set, with an arc from $x$ to $y$ if $y$ is a power of $x$. It is known that, for finite groups, the power graph determines the directed power graph up to isomorphism. However, it is not true that any isomorphism between power graphs induces an isomorphism between directed power graphs. Moreover, for infinite groups the power graph may fail to determine the directed power graph. In this paper, we consider power graphs of torsion-free groups. Our main results are that, for torsion-free nilpotent groups of class at most 2 , and for groups in which every non-identity element lies in a unique maximal cyclic subgroup, the power graph determines the directed power graph up to isomorphism. For specific groups such as $\mathbb{Z}$ and $\mathbb{Q}$, we obtain more precise results. Any isomorphism $P(\mathbb{Z}) \rightarrow P(G)$ preserves orientation, so induces an isomorphism between directed power graphs; in the case of $\mathbb{Q}$, the orientations are either all preserved or all reversed. We also obtain results about groups in which every element is contained in a unique maximal cyclic subgroup (this class includes the free and free abelian groups), and about subgroups of the additive group of $\mathbb{Q}$ and about $\mathbb{Q}^{n}$.
\end{abstract}

Keywords Power graph · Directed power graph · Torsion-free group

Horacio Guerra and Šimon Jurina acknowledge funding from the School of Mathematics and Statistics for summer internships during which this research was carried out.

$凶$ Peter J. Cameron

pjc20@st-andrews.ac.uk

1 University of St Andrews, North Haugh, St Andrews, Fife KY16 9SS, UK

2 Present Address: School of Mathematics and Statistics, Newcastle upon Tyne NE1 7RU, UK 
Mathematics Subject Classification 05C25 - 20F99

\section{Introduction}

Let $G$ be a group. Then the power graph of $G$ is the graph $P(G)$ with vertex set $V(P(G))=G$ and edge set

$$
E(P(G))=\left\{\{x, y\}:(\exists n \in \mathbb{Z} \backslash\{0\})\left(x=y^{n} \text { or } y=x^{n}\right)\right\} .
$$

The directed power graph of $G$ is the directed graph $\vec{P}(G)$ with vertex set $V(\vec{P}(G))=G$ and arc set

$$
E(\vec{P}(G))=\left\{(x, y):(\exists n \in \mathbb{Z} \backslash\{0\})\left(y=x^{n}\right)\right\}
$$

Thus, $\vec{P}(G)$ is an orientation of $P(G)$.

When $x$ and $y$ are connected in $P(G)$, we write $x \sim y$. If $a$ is a power of $b$ in $G$, we denote this by $b \rightarrow a$.

In a graph $\Gamma$, we denote the set of neighbours of a vertex $x$ by $N(x)$; in a directed graph, we denote the set of in-neighbours of $x$ by $I(x)$, and the set of out-neighbours by $O(x)$. Since we will always be considering power graphs of groups, we denote $N_{G}(x)$ for the set of neighbours of $x$ in $P(G)$, and $I_{G}(x), O_{G}(x)$ for the sets of inand out-neighbours of $x$ in $\vec{P}(G)$.

The directed power graph was first defined in the context of semigroups by Kelarev and Quinn [6]; the undirected power graph by Chakrabarty et al. [5]. The first author [3] showed the following result:

Theorem 1.1 Let $G$ and $H$ be finite groups such that $P(G) \cong P(H)$. Then $\vec{P}(G) \cong$ $\vec{P}(H)$.

However, it is not true that any isomorphism from $P(G)$ to $P(H)$ preserves orientations of edges; and the theorem above fails for infinite groups.

Example Let $G$ be the cyclic group of order 6, generated by $a$. Then $P(G)$ is the complete graph $K_{6}$ with the two edges $\left\{a^{2}, a^{3}\right\}$ and $\left\{a^{4}, a^{3}\right\}$ removed. So its automorphism group permutes $\left\{1, a, a^{5}\right\}$ transitively; the power graph does not determine the identity uniquely.

Example (from [4]) Let $G$ be the Prüfer group $\mathbb{Z}_{p^{\infty}}$, defined as the quotient $\mathbb{L}_{p} / \mathbb{Z}$, where $\mathbb{L}_{p}$ is the set of rationals with $p$-power denominators (where $p$ is prime). Then every element of $G$ has $p$-power order, and every proper subgroup is a finite cyclic group; so $P(G)$ is a countable complete graph. Thus, knowledge of $P(G)$ does not even determine the prime $p$.

In this paper, we consider torsion-free groups, to avoid difficulties suggested by the above examples. Our main results are the following theorems. 
Theorem 1.2 Let $H$ be a group with $P(H)$ isomorphic to $P(\mathbb{Z})$. Then $H$ is isomorphic to $\mathbb{Z}$, and any isomorphism from $P(\mathbb{Z})$ to $P(H)$ induces an isomorphism from $\vec{P}(\mathbb{Z})$ to $\vec{P}(H)$.

Theorem 1.3 Let $G$ and $H$ be nilpotency class 2 torsion-free groups. Then $P(G) \cong$ $P(H)$ implies $\vec{P}(G) \cong \vec{P}(H)$.

We will see examples showing that, under these hypotheses, it is not true that $P(G) \cong P(H)$ implies $G \cong H$ and also examples to show that some hypothesis on $G$ is needed.

Theorem 1.4 Let $G$ be a torsion-free group in which any non-identity element lies in a unique maximal cyclic subgroup. Then, for any group $H$, any isomorphism from $P(G)$ to $P(H)$ induces an isomorphism from $\vec{P}(G)$ to $\vec{P}(H)$.

The class of groups covered by this theorem includes direct sums of copies of the additive group of $\mathbb{Z}$, free groups, and indeed any torsion-free nilpotent group of class 2 in which every element is contained in some maximal cyclic subgroup (see Proposition 5.3). Our result about such groups is stronger than indicated, see Theorem 5.4.

Theorem 1.5 Let $\mathbb{Q}$ be the additive group of rational numbers, and $G=\mathbb{Q}^{n}$. Then, for a group $H$, if $P(G) \cong P(H)$, then $\vec{P}(G) \cong \vec{P}(H)$. Moreover, if $n=1$, then any isomorphism from $P(G)$ to $P(H)$ either preserves or reverses the orientation of edges.

We also include some detailed results about power graphs of subgroups of $\mathbb{Q}$, where we exhibit non-isomorphic subgroups with isomorphic power graphs.

We note that further investigation of the power graph can be found in [1]; this and other papers concentrate on graph-theoretic aspects.

\section{On the definition}

We have excluded $n=0$ in the definition of edges in the power graph and the directed power graph. We make some brief comments on this. The alternative definition would ensure that there is an edge from every vertex $x$ to $x^{0}=1$ in the directed power graph.

If $G$ is a torsion group (in particular, if $G$ is finite), then this makes no difference at all, since our definition as stated gives an edge from $x$ to 1 if $x^{n}=1$ for some $n>0$.

We are concerned here with torsion-free groups. A group $G$ is torsion-free if every non-identity element has infinite order. Note that, if $G$ is torsion-free and $a \in G$ satisfies $a^{m}=a^{n}$ where $m \neq n$, then $a^{m-n}=1$, so $a=1$.

For a torsion-free group, with the definition modified to allow $n=0$, the identity is the unique sink in the directed power graph (there is an arc from every vertex to it), and so we can uniquely identify it. The situation in the undirected power graph is a little different:

Proposition 2.1 Let $x$ be an element of the torsion-free group $G$. Then $x$ is joined to every vertex in the undirected power graph (including edges from $x$ to $x^{0}$ ) if and only if one of the following holds: 
- $x$ is the identity;

- $G$ is the infinite cyclic group and $x$ is a generator.

Proof The sufficiency is clear. So suppose that, for every $y \in G$, either $y=x^{n}$ or $x=y^{n}$ for some integer $n$, but $x$ is neither the identity nor a generator. Note that $n$ is unique in either case. Since $x$ is not a generator, there exists $y$ such that $x=y^{n}$ for some $n>1$. Choose an integer $m>1$ coprime to $n$ and let $z=y^{m}$. If $x=z^{k}$, then $y^{n}=y^{m k}$, so $n=m k$, implying that $m \mid n$; if $z=x^{k}$, then $y^{n k}=y^{m}$, whence $n k=m$, and $n \mid m$. Either statement contradicts $\operatorname{gcd}(n, m)=1$.

Thus, if $G$ is not infinite cyclic, we can recognize the identity. In the case where $G=\mathbb{Z}$, the identity and the two generators are indistinguishable in the power graph and are permuted transitively by its automorphism group, so we can choose any one to be the identity and delete the edges containing it to get a graph isomorphic to the power graph as defined in this paper.

So our theorems would be essentially unaffected by changing the definition. We use the definition given because it makes some of the arguments simpler.

\section{Preliminary results}

We collect here a few lemmas of general use.

Lemma 3.1 Let $G$ be a group with $P(G)$ having exactly one isolated vertex. Then $G$ is torsion-free.

Proof Since $P(G)$ has a unique isolated vertex, it suffices then to show that this must be the identity of $G$. Let $a \in G$ be non-identity. If $a=a^{-1}$, we have $a^{2}=1_{G}$, so $a$ is not the isolated vertex. On the other hand, if $a \neq a^{-1}$, then $a$ and $a^{-1}$ are joined, so again $a$ is not isolated.

For $a, b \in G$ define

$$
S_{a, b}:=\{c \in G: c \sim b \text { and } c \nsim a\} .
$$

Lemma 3.2 Let $G$ be a group with $P(G)$ having exactly one isolated vertex, and suppose that $a, b \in G$ with $a \sim b$. Then $S_{a, b}=S_{b, a}=\varnothing$ if and only if $a=b^{ \pm 1}$.

Proof $(\Leftarrow)$ Observe that $a=b^{ \pm 1}$ implies that $a$ and $b$ have the same neighbours in the power graph.

$(\Rightarrow)$ We have that $a \sim b$. If either is the identity then so is the other, so we are done. Hence, we have that $a$ and $b$ are non-identity.

Suppose first that $a=b^{m}$ for some $m \in \mathbb{Z}$. If $|m|>1$, then choose $j>1$ such that $\operatorname{gcd}(j, m)=1$. We claim $b^{j} \nsim a$. Indeed, suppose that $a=b^{j t}$, for some $t \in \mathbb{Z}$. Then $b^{m}=b^{j t}$, so by Lemma 3.1 and our earlier remark, we deduce that $m=j t$, so $\operatorname{gcd}(j, m)=j>1$, a contradiction.

Otherwise, suppose $b^{j}=a^{t}$ for some $t \in \mathbb{Z}$. We deduce that $b^{j}=b^{m t}$, so by Lemma 3.1 we have $j=m t$, so $\operatorname{gcd}(j, m)=|m|>1$, also a contradiction. 
Thus, $b^{j} \nsim a$, and since there are infinitely many choices for $j$, all giving pairwise distinct elements $b^{j}$, we have that $S_{a, b}$ is infinite. Therefore, we must have $|m|=1$, so $a=b^{ \pm 1}$.

Similarly, if $a^{m}=b$ for some $m \in \mathbb{Z}$, a symmetric argument shows $m= \pm 1$.

Note that the proof shows that, if $b \rightarrow a$ but $a \nrightarrow \rightarrow b$, then $S_{a, b}$ is infinite.

From now on, when dealing with a torsion-free group, we will use without mention the result above: we can always recognize inverse elements in the power graph of the group.

We conclude this section with a couple more results which will be needed later.

Lemma 3.3 Let $G$ be a torsion-free group and $x$ a non-identity element of $G$. Then the induced subgraph of $P(G)^{\prime}$ on $O(x)=O_{G}(x)$ is a connected subgraph of $P(G)^{\prime}$, and there are no edges between $I(x)$ and $O(x)$ in $P(G)^{\prime}$.

Proof Let $x^{m}$ and $x^{n}$ be in $O(x)$. Then if $p$ is a prime dividing neither $m$ nor $n$, then $x^{p}$ is joined to both $x^{m}$ and $x^{n}$ in the complement of the power graph.

Finally, if we have $y \rightarrow x \rightarrow z$, then $y \rightarrow z$. Thus, no in-neighbour is connected to an out-neighbour in the complement of the power graph.

Lemma 3.4 Let $G$ be a torsion-free group and $H$ be a group with $P(G) \cong P(H)$. Fix $z \in G$ such that $z \neq 1_{G}$ and let $f$ be an isomorphism $f: P(G) \rightarrow P(H)$. Then $f$ induces an isomorphism from each connected component of $P(G)^{\prime}$ in $N_{G}(z)$ to a connected component of $P(H)^{\prime}$ in $N_{H}(f(z))$. Furthermore, $I_{G}(z) \cong I_{H}(f(z))$ and $O_{G}(z) \cong O_{H}(f(z))$.

Proof By Lemma 3.1, $H$ is torsion-free and $f(z) \neq 1_{H}$. The lemma will follow from the next result:

Claim For all connected components $C$ of $N(z)^{\prime}$ and $x, y \in C, f(x)$ and $f(y)$ belong to the same connected component $D$ of $N(f(z))^{\prime}$. (Here we use $N(z)^{\prime}$ for the induced subgraph of $P(G)^{\prime}$ on $N(z)$.)

To verify the claim, suppose that there exists a connected component $C$ of $N(z)^{\prime}$ and $x, y \in C$ such that $f(x) \in D_{1}$ and $f(y) \in D_{2}$, where $D_{1}$ and $D_{2}$ are different connected components of $N(f(z))^{\prime}$. Then there exists a path $\left(x_{0}=x, x_{1}, \ldots, x_{n}=y\right)$ in $C$. As $f$ is an isomorphism from $P(G)$ to $P(H)$, it follows that there is a path $\left(f\left(x_{0}=x\right), f\left(x_{1}\right), \ldots, f\left(x_{n}=y\right)\right)$ in $P(H)^{\prime}$ and $f(N(z))=N(f(z))$. Hence, for all $i \in\{0,1, \ldots, n\}$, we have $f\left(x_{i}\right) \in N(f(z))^{\prime}$. But this is a contradiction as $f(x)$ and $f(y)$ belong to different connected components of $N(f(z))^{\prime}$.

We are ready to prove the lemma. As $G$ and $H$ are torsion-free, we have $O(x)=$ $\left\{x^{n}: n \in \mathbb{Z} \backslash\{0\}\right\}$, and so $O(f(x)) \cong P(\mathbb{Z}) \backslash\{0\} \cong O(x)$. Furthermore, as $f$ is an isomorphism, using our claim, we deduce that $f$ induces an isomorphism from each connected component of $N(z)^{\prime}$ to a connected component of $N(f(z))^{\prime}$. It remains to show that $I(z) \cong I(f(z))$. By Lemma 3.3, we have to consider two cases, either $f(O(z))=O(f(z))$ or $f(O(z))=D$, where $D$ is a connected component of $I(f(z))^{\prime}$. In the first case, $f$ induces an isomorphism from each connected component of $I(z)^{\prime}$ to a connected component of $I(f(z))^{\prime}$. In the second case, using our 
results, there exists a connected component $C$ of $I(z)^{\prime}$ such that $f(C)=O(f(z))^{\prime}$. Hence, there are two connected components in both $N(z)^{\prime}$ and $N(f(z))^{\prime}$ isomorphic to $P(\mathbb{Z}) \backslash\{0\}$. Then $f$ induces an isomorphism from each of the remaining connected components of $I(z)^{\prime}$ to one of the remaining connected components of $I(f(z))^{\prime}$. Putting this together, we deduce that in both cases $I(z) \cong I(f(z))$.

\section{The group $\mathbb{Z}$}

In this section, we examine the power graph of $\mathbb{Z}$.

Lemma 4.1 Let $a, b \in \mathbb{Z}$ be such that $a \sim b$ and $a \neq \pm b$. Then $a \rightarrow b$ if and only if $S_{a, b}$ is finite.

Proof $(\Rightarrow)$ Notice that $b$ is only divisible by finitely many $c \in \mathbb{Z}$. On the other hand, if $b$ divides $x$ then $a$ divides $x$. Thus, there are at most finitely many vertices that are connected to $b$ but not to $a$, that is, $S_{a, b}$ is finite.

$(\Leftarrow)$ By Lemma 4.1, we know that if $a \not t b$, then $b \rightarrow a$, so $S_{a, b}$ is infinite.

This shows that the undirected power graph of $\mathbb{Z}$ determines the directed power graph, by the rule in the Lemma. Using this, we prove Theorem 1.2.

Proof of Theorem 1.2 Let $G=\mathbb{Z}$. We note first that $P(H)$ has an isolated vertex, so $H$ is torsion-free. Putting Lemmas 3.2 and 4.1 together we observe that if $a \sim b$, $a \neq b$ in $P(G)$, then one of the following holds:

(a) $S_{a, b}=S_{b, a}=\varnothing$;

(b) one of $S_{a, b}$ or $S_{b, a}$ is finite and the other is infinite.

Therefore, the same holds in $P(H)$. So consider $a \sim b$ in $P(H)$ with $a \neq b$. If we are in the first case, then, by Lemma 3.2, we deduce that $a=b^{-1}$, so $\vec{P}(H)$ has directed arrows going in both directions. But the same is true in $\vec{P}(G)$, so for all these cases the directions agree. If we are in the second case, say $S_{a, b}$ is finite and $S_{b, a}$ is infinite, then the corresponding elements of $G$, say $a^{\prime}$ and $b^{\prime}$, have a directed edge in $\vec{P}(G)$ going from $a^{\prime}$ to $b^{\prime}$, but not the other way around. Suppose that the direction in $\vec{P}(H)$ was reversed, so that $a=b^{m}$ for some $m \in \mathbb{Z}$. The argument in Lemma 3.2 shows that $|m|>1$ implies that $S_{a, b}$ is infinite, contrary to our assumption. Thus, we must have $a=b^{-1}$, but this is also contrary to our assumption that $S_{b, a}$ is infinite. Thus, we must have the directions agreeing in the power graph of $G$ for the second case as well.

Now, if $G=\langle a\rangle$, then there is a directed arrow from $a$ to every other element of $G$ except the identity. So $H$ has such a vertex also, and $H$ is an infinite cyclic group, as needed.

Remark If we had used the alternative definition of the power graph, where $x$ is joined also to $x^{0}=1$, then it is false that any isomorphism of the power graph induces an isomorphism of the directed power graph, since as noted earlier the identity and the two generators are indistinguishable in the power graph. We can conclude that, with this definition, if $P(H) \cong P(\mathbb{Z})$, then $\vec{P}(H) \cong \vec{P}(\mathbb{Z})$. 


\section{Groups with the same power graph as $\mathbb{Z}^{n}$}

One may be tempted to conjecture that, for all $n \in \mathbb{N}$, the power graph of $\mathbb{Z}^{n}$ determines $\mathbb{Z}^{n}$ up to isomorphism, as we showed was true for $\mathbb{Z}$ in Theorem 1.2. However, this is not the case. In fact, we will prove that, for $n>1$, all the groups $\mathbb{Z}^{n}$ have isomorphic power graphs.

In this section, we are interested in a wider class of groups, namely those with the following property $(*)$ :

Every non-identity element is contained in a unique maximal cyclic subgroup.

We begin with a few remarks about this class. First, observe that the property is equivalent to saying that the non-identity elements of the group are partitioned into maximal cyclic subgroups. Hence,

Proposition 5.1 Let $G$ be a torsion-free group in which every non-identity element is contained in a unique maximal cyclic subgroup. Then the power graph of $G$ is the disjoint union of an isolated vertex (the identity) and a number of copies of $P(\mathbb{Z}) \backslash\left\{1_{\mathbb{Z}}\right\}$.

How many connected components are there? This is answered by the next result.

Proposition 5.2 Let $G$ be a torsion-free group in which every non-identity element lies in a unique maximal infinite cyclic subgroup. Then the number of maximal infinite cyclic subgroups is either 1 or infinite. In particular, if $G$ is countable but not isomorphic to $\mathbb{Z}$, then the number of such subgroups is countably infinite.

Proof Suppose, for a contradiction, that $a_{1}, \ldots, a_{k}$ are all the generators for the maximal infinite cyclic subgroups of $G$, where $k>2$. (Each such subgroup has two generators, which are inverses of each other.) By hypothesis, $G=\left\langle a_{1}, \ldots, a_{k}\right\rangle$.

Now $G$ acts on itself by conjugation; this action must map the set

$$
\left\{a_{1}, \ldots, a_{k}\right\}
$$

into itself and so induces a subgroup of the symmetric group $S_{k}$ on this set. The kernel of this action is a subgroup $H$ of finite index in $G$ which fixes all of $a_{1}, \ldots, a_{k}$; we see that $H$ is the centre $Z(G)$ of $G$, and so $H$ is abelian.

Now $H$ is an infinite abelian group which is partitioned by its intersections with the maximal cyclic subgroups of $G$. But if $a$ and $b$ are elements of $H$ belonging to distinct such subgroups, then $\langle a, b\rangle \cong \mathbb{Z}^{2}$, and this group cannot be covered by finitely many cyclic subgroups.

Which groups have this property? One class is given by the next result.

Proposition 5.3 Let $G$ be a torsion-free group of nilpotency class 2 , and suppose a and $b$ generate distinct maximal infinite cyclic subgroups. Then $\langle a\rangle \cap\langle b\rangle=\{1\}$.

Proof Suppose that $\langle a\rangle \cap\langle b\rangle=\langle x\rangle$, for some $x \in G, x \neq 1$. So $x=a^{m}=b^{n}$ for some $n, m \in \mathbb{Z}$. Then, since $a^{m}$ is a power of $b$, we have $1=\left[a^{m}, b\right]$. Since $\left[a_{1} a_{2}, b\right]=\left[a_{1}, b\right]\left[a_{2}, b\right]$ in a nilpotent group of class 2 , we have $1=[a, b]^{m}$. But, since $G$ is torsion-free, we have $[a, b]=1$, so $\langle a, b\rangle$ is abelian. 
So this subgroup is equal to one of $\mathbb{Z}^{2}$, or $\mathbb{Z} \times C_{k}$ for some natural number $k$. It cannot be $\mathbb{Z} \times C_{k}$ for any $k>1$, as then $G$ would not be torsion-free. Also, it cannot be $\mathbb{Z}$, since $\langle a\rangle$ and $\langle b\rangle$ are maximal cyclic, so this would force $\langle a, b\rangle=\langle a\rangle=\langle b\rangle$, contrary to our assumption. Finally, we observe that $\langle a, b\rangle /\langle a\rangle$ is finite, but $\mathbb{Z}^{2} /\langle g\rangle$ is infinite for any $g \in \mathbb{Z}^{2}$. (This is clear if $g$ is the identity, so suppose not. Let $g=(m, n)$ where, without loss of generality, $n \neq 0$. The elements $(k, 0)$ for $k \in \mathbb{Z}$ all lie in different cosets of $\langle g\rangle$.) Hence, $\langle a\rangle \cap\langle b\rangle=\{1\}$, as needed.

This shows, for example, that $\mathbb{Z}^{n}$ has property $(*)$ for finite $n$. It is enough to prove that each non-identity element of $\mathbb{Z}^{n}$ lies in a maximal cyclic subgroup. The element $\left(a_{1}, \ldots, a_{n}\right)$, with $a_{i}$ not all zero, lies in the maximal cyclic subgroup $\left\langle\left(a_{1} / d, \ldots, a_{n} / d\right)\right\rangle$, where $d=\operatorname{gcd}\left(a_{1}, \ldots, a_{n}\right)$.

Other groups with this property include free groups.

Remark Consider the two conditions on a torsion-free group $G$ :

(a) every non-identity element lies in a maximal cyclic subgroup;

(b) every non-identity element lies in a unique maximal cyclic subgroup.

Now (a) does not imply (b) in general. For take the group generated by $a$ and $b$ with the single defining relation $a^{m}=b^{n}$ where $m, n>1$. This group is a free product with amalgamation $A *_{C} B$, where $A$ and $B$ are the groups generated by $a$ and $b$, respectively, and $C$ is generated by $a^{m}=b^{n}$. The theory of such groups tells us [9]:

- $A$ and $B$ embed into $A *_{C} B$;

- any element which is not in a conjugate of $A$ or $B$ has infinite order.

It follows that the group is torsion-free. Clearly, the element $a^{m}=b^{n}$ lies in two distinct maximal cyclic subgroups. On the other hand, by Proposition 5.3, in torsionfree abelian groups, or nilpotent groups of class 2, (a) does imply (b).

For groups with property $(*)$, we can make a strong statement about the power graphs.

Theorem 5.4 Let G be a countable torsion-free group which is not cyclic, but in which each non-identity element lies in a unique maximal cyclic subgroup. Let $H$ be a group with $P(H) \cong P(G)$. Then

(a) each non-identity element of $H$ lies in a unique maximal cyclic subgroup;

(b) $\vec{P}(H) \cong \vec{P}(G)$;

(c) any isomorphism from $P(G)$ to $P(H)$ induces an isomorphism from $\vec{P}(G)$ to $\vec{P}(H)$.

Moreover, all groups $G$ satisfying the hypothesis have isomorphic power graphs.

Proof For a group satisfying the hypotheses of the theorem, there are countably many connected components of $P(G)$ (with the identity removed), each isomorphic to $P(\mathbb{Z})$ with the identity removed. So the last statement holds. Since the power graph of $P(\mathbb{Z})$ determines the directions on edges (Lemma 4.1), (b) and (c) hold.

Now suppose that $f: \vec{P}(G) \rightarrow \vec{P}(H)$ is a directed power graph isomorphism. Then each connected component of $\vec{P}(H)$ has a vertex $a$ with an arc to all other 
vertices of the component (the image under $f$ of a generator of a maximal cyclic subgroup of $G$ ); so the component together with the identity is a maximal cyclic subgroup. This proves (a).

Corollary 5.5 The groups $\mathbb{Z}^{n}$, for $n \in \mathbb{N}, n \geq 2$, or the direct sum of countably many copies of $\mathbb{Z}$, all have isomorphic (directed) power graphs.

\section{The groups $\mathbb{Q}$ and $\mathbb{Q}^{n}$}

Next turn to study the additive group of the rationals. Before proving the main theorem, we prove an auxiliary lemma. As before, if $a$ is a vertex of a directed graph, let $I(a)$ and $O(a)$ denote the sets of in-neighbours and out-neighbours of $a$.

Lemma 6.1 For $a \in \mathbb{Q} \backslash\{0\}$, define the map $\varphi_{a}: \mathbb{Q} \rightarrow \mathbb{Q}$ by $x \mapsto a^{2} / x$ and $0 \mapsto 0$. Then $\varphi_{a}$ is an automorphism of $P(\mathbb{Q})$ and an isomorphism from $\vec{P}(\mathbb{Q})$ to the directed power graph of $\mathbb{Q}$ with all arrows reversed. Furthermore, it is an isomorphism from $O(a)$ to $I(a)$.

Proof It is straightforward to verify that $\varphi_{a}$ is a bijection. If $x \sim y$, then we have $x=n y$ for some $n \in \mathbb{Z}$, say. Then $a^{2} / x=a^{2} / n y$, so $\varphi(y)=n \varphi(x)$, so $\varphi_{a}(x) \sim \varphi_{a}(y)$. We have $x \rightarrow y$ if, and only if, $\varphi_{a}(y) \rightarrow \varphi_{a}(x)$, if, and only if, $\varphi_{a}(x) \rightarrow \varphi_{a}(y)$ in the reversed power graph, as needed.

We have

$$
\begin{aligned}
\varphi_{a}(O(a)) & =\{\varphi(n a): n \in \mathbb{Z}\} \\
& =\{a / n: n \in \mathbb{Z}\} \\
& =I(a),
\end{aligned}
$$

so $\varphi_{a}$ maps $O(a)$ to $I(a)$ bijectively and preserves edge relationships, as required.

Now we can prove part of Theorem 1.5.

Theorem 6.2 Let $G$ be a group with $P(G) \cong P(\mathbb{Q})$. Then $\vec{P}(G) \cong \vec{P}(\mathbb{Q})$.

Proof Let $x \in \mathbb{Q}$ be non-identity. Then by Lemma 6.1, we have that $O(x) \cong I(x)$, so $O(x)^{\prime} \cong I(x)^{\prime}$. Let $g \in G$ be non-identity. Then by Lemma 3.3 we have that $I(g)^{\prime}$ and $O(g)^{\prime}$ have no edges between them. So the complement of the power graph of $G$ restricted to $N(\mathrm{~g})$ consists of two components, one of which is connected. By Lemma 3.4, an isomorphism $f: P(\mathbb{Q}) \rightarrow P(G)$ must map $I(x)$ to either $I(f(x))$ or $O(f(x))$, since $N(x)^{\prime}$ and $N(f(x))^{\prime}$ have the same number of connected components. Similarly, $f(O(x))=O(f(x))$ or $f(O(x))=I(f(x))$.

We can now show our result. Suppose that for $x, y \in \mathbb{Q}$, we have $x \rightarrow y$. If $f(x) \rightarrow f(y)$, then we claim that $\vec{P}(G) \cong \vec{P}(\mathbb{Q})$. We know that $f(O(x))=$ $O(f(x))$ and similarly $f(I(x))=I(f(x))$. If $y \sim z$, then the direction agrees with $f(y) \sim f(z)$, since $f(x) \in I(f(y))$ imply $f(I(y))=I(f(y))$ and similarly for $O(y)$. Repeating this procedure, we can deduce that the directions of any path 
$\left(f(x)=f\left(x_{1}\right), f\left(x_{2}\right), \ldots, f\left(x_{n}\right)\right)$ agree with those of the corresponding path $(x=$ $\left.x_{1}, x_{2}, \ldots, x_{n}\right)$. Since the graph $P(\mathbb{Q})$ is connected we can reach any point in $P(G)$ by a path starting at $f(x)$.

Now suppose that $f(y) \rightarrow f(x)$ instead. Then we have $f(I(x))=O(f(x))$ and $f(O(x))=I(f(x))$. Again, if $y \sim z$, then the direction disagrees with $f(y) \sim f(z)$, since $f(x) \in O(f(y))$ implies $f(I(y))=O(f(y))$ and similarly for $O(y)$. Repeating this procedure, we can deduce that the directions of any path $\left(f(x)=f\left(x_{1}\right), f\left(x_{2}\right), \ldots, f\left(x_{n}\right)\right)$ are in exact reversal with respect to those of the corresponding path $\left(x=x_{1}, x_{2}, \ldots, x_{n}\right)$. Thus, $\vec{P}(G)$ has all the arrows reversed relative to $\vec{P}(\mathbb{Q})$, so we deduce that $\vec{P}(G) \cong \vec{P}(\mathbb{Q})$.

We turn now to the group $\mathbb{Q}^{n}$ for $n>1$, and prove the remaining part of Theorem 1.5.

If $a$ and $b$ are non-identity elements, then $a$ and $b$ lie in the same connected component of the power graph if and only if they span the same 1-dimensional vector subspace of $\mathbb{Q}^{n}$. (For, if $x$ and $y$ lie in the same vector subspace, then $y=(m / n) x$ for some $m, n \in \mathbb{Z}$, so $n y=m x$, and there is a path of length 2 from $x$ to $y$. The converse is clear.)

So the power graph of $\mathbb{Q}^{n}$ consists of countably many disjoint copies of $P(\mathbb{Q}) \backslash\{0\}$ together with an isolated vertex. For $x \neq 0$, let $Q_{x}$ denote the connected component containing $x$.

Theorem 6.3 Let $G$ be a group with $P(G) \cong P\left(\mathbb{Q}^{n}\right)$. Then $\vec{P}(G) \cong \vec{P}\left(\mathbb{Q}^{n}\right)$.

Proof Let $f: \mathbb{Q}^{n} \rightarrow G$ be a power graph isomorphism. Let $x \in \mathbb{Q}^{n}$ be non-identity. We deduce from Lemma 6.1 that $I(x) \cong O(x)$. By Lemma 3.1, $G$ must be torsionfree, so we can apply Lemma 3.3 to deduce, by the same arguments as in the proof of Theorem 6.2, that $O(f(x)) \cong I(f(x))$ (since $f(I(x))=I(f(x))$ or $f(I(x))=$ $O(f(x))$ and similarly for $O(x))$ and thus that the connected component containing $f(x), C_{f(x)}$, has a directed power graph isomorphic to that of the connected component $Q_{x}$ containing $x$. Repeating this procedure for all the connected components of $G$, we conclude that $\vec{P}(G) \cong \vec{P}\left(\mathbb{Q}^{n}\right)$.

\section{Subgroups of $\mathbb{Q}$}

We now examine the power graphs of subgroups of $\mathbb{Q}$. We begin with a general result.

Lemma 7.1 Let $G$ be a nilpotency class 2 torsion-free group and $C$ a connected component of $P(G)$. Then the vertices of $C$ form a subgroup of $G$.

Proof We first show that $x$ and $y$ in $C$ being two steps apart implies that $\langle x, y\rangle$ is cyclic. If we have any of the possibilities

$$
\begin{aligned}
& z \rightarrow x, z \rightarrow y: \text { then } x, y \in\langle z\rangle \\
& z \rightarrow x, y \rightarrow z: \text { then } x \in\langle y\rangle \\
& z \rightarrow y, x \rightarrow z: \text { similar; }
\end{aligned}
$$


then we are done. Hence, suppose that $x \rightarrow z$ and $y \rightarrow z$, so $z=x^{n}=y^{m}$ for some $n, m \in \mathbb{Z}$. By the same argument as in Proposition 5.3, we see that $\langle x, y\rangle$ is a finitely generated abelian group, it must be one of $\mathbb{Z}^{2}$ or $\mathbb{Z} \times C_{k}$. Since $G$ is torsion-free, it cannot be $\mathbb{Z} \times C_{k}$ for $k>1$. It cannot be $\mathbb{Z}^{2}$, since $\langle x\rangle$ is a cyclic subgroup of finite index, contradicting the result in Proposition 5.3. Therefore, $\langle x, y\rangle \cong \mathbb{Z}$.

Now we show that for all $x, y \in C,\langle x, y\rangle$ is cyclic. We use induction on the length of the path from $x$ to $y$. Suppose that $z$ is the point on the path two steps from $x$. Then $\langle x, z\rangle=\langle w\rangle$, and the path from $w$ to $y$ is shorter than the path from $x$ to $y$; so $\langle w, y\rangle$ is cyclic and contains $x$.

Finally, let $x, y \in C$. We have $\langle x, y\rangle=\langle a\rangle$ for some $a \in C$. Then $x y^{-1} \in\langle a\rangle \subseteq C$, so $x y^{-1} \in C$. Thus, $C \leq G$, as claimed.

Before we continue, we state a result which can be found in [8].

We define a unitary subgroup of $\mathbb{Q}$ to be a subgroup that contains 1 .

Theorem 7.2 Every non-trivial subgroup $S$ of $\mathbb{Q}$ is isomorphic to at least one unitary subgroup of $\mathbb{Q}$.

Define $P$ to be the set of all prime numbers and let $M$ be the set $\{f: P \rightarrow$ $\{\mathbb{N} \cup\{0, \infty\}\}$. Any $f \in M$ is called a height function.

Definition For a unitary subgroup $A$ of $\mathbb{Q}$, the height function $h_{A} \in M$ associated to $A$ is defined as follows: for each prime $p, h_{A}(p)=\max \left\{\alpha: \frac{1}{p^{\alpha}} \in A\right\}$.

Next we state two results of [8].

Lemma 7.3 Let $A$ be a unitary subgroup of $\mathbb{Q}$. Then for relatively prime $m$ and $n$, $m / n \in A$ if, and only if, $1 / n \in A$.

Lemma 7.4 Let $A$ be a unitary subgroup of $\mathbb{Q}$. Then for relatively prime $m$ and $n$, $1 /(m n) \in A$ if, and only if, $1 / m \in A$ and $1 / n \in A$.

We are now ready to prove several auxiliary results. In what follows, we work in the power graph $P(A)$ but take restrictions to the set $I_{A}(x)$ of in-neighbours of a vertex in the directed power graph $\vec{P}(A)$.

Lemma 7.5 Let $A$ be a unitary subgroup of $\mathbb{Q}$ and $P(A)$ be the power graph of $A$. Then there exists $x \in A$ such that the set $I_{A}(x)$ of in-neighbours of $x$ in $P(A)$ is infinite if, and only if, either there exists a prime $p$ such that $h_{A}(p)=\infty$, or there are infinitely many primes $q$ such that $h_{A}(q)>0$.

Proof If there exists a prime $p$ such that $h_{A}(p)=\infty$ or there are infinitely many primes $q$ such that $h_{A}(q)>0$, then $I_{A}(1)$ is infinite.

In order to prove the forward implication, we will prove the contrapositive. Let $x \in A$. If $x=0$, then clearly $I_{A}(x)$ is finite, so suppose $x \neq 0$. If $y \in I_{A}(x)$, then $|y|<|x|$. Let $|y|=m / n$, where $\operatorname{gcd}(m, n)=1$. Factorize $n$ to the form

$$
n= \pm p_{1}^{\alpha_{1}} p_{2}^{\alpha_{2}} \cdots p_{n}^{\alpha_{n}}
$$


where each $p_{i}$ is a prime and $\alpha_{i} \in \mathbb{N}$. By Lemma 7.3, $m / n \in A$ if, and only if, $1 / n \in A$. Hence, by repeatedly using Lemma $7.4, m / n \in A$ if, and only if,

$$
\frac{1}{p_{i}^{\alpha_{i}}} \in A
$$

for all $i \in 1,2, \ldots, n$. By our assumption, there are only finitely many numbers in $A$ of the form $\frac{1}{p^{\alpha}}$, where $p$ is a prime and $\alpha \in \mathbb{N}$; hence, there are only finitely many possibilities for the value of $n$ as $y$ ranges over $I_{A}(x)$. For fixed $n$, since $|m|<|x| \cdot|n|$, there are only finitely many possibilities for $m$. We conclude that $I_{A}(x)$ is finite.

Lemma 7.6 Let $A$ be a unitary subgroup of $\mathbb{Q}$ and $P(A)$ be the power graph of $A$. Then the following two statements are equivalent:

- There exists $x \in A$ such that $I_{A}(x)$ is infinite.

- For all $x \in A$ such that $x \neq 0, I_{A}(x)$ is infinite.

Proof Observe that the converse implication is trivial. So suppose there exists $x \in A$ such that $I_{A}(x)$ is infinite. Then by Lemma 7.5, there exists a prime $p$ such that $h_{A}(p)=\infty$ or there are infinitely many primes $q$ such that $h_{A}(q)>0$. Suppose there exists a prime $p$ such that $h_{A}(p)=\infty$. Let $y=m / n \in A$ be non-identity. Since $I_{A}(y)=I_{A}(-y)$ we can assume without loss of generality that $y>0$. Moreover, we can assume $\operatorname{gcd}(m, n)=1$. By Lemma 7.3, $m / n \in A$ if, and only if, $1 / n \in A$. If $\operatorname{gcd}(p, n)=1$, then using Lemma 7.4 , we have that $1 /\left(n p^{\alpha}\right) \in A$ for all $\alpha \in \mathbb{N}$. Let $\operatorname{gcd}(p, m)=p^{\beta}$ and $m=k p^{\beta}$, for some $k \in \mathbb{Z}$.

Since $\operatorname{gcd}(n, k)=1$ and $\operatorname{gcd}(p, k)=1$, we have by Lemma 7.3,

$$
\frac{k}{n p^{\alpha}}=\frac{k p^{\beta}}{n p^{\alpha+\beta}}=\frac{m}{n p^{\alpha+\beta}} \in A
$$

for all $\alpha \in \mathbb{N}$. Hence, $I_{A}(y)$ is infinite. If $\operatorname{gcd}(p, n) \neq 1$, then $\operatorname{gcd}(p, m)=1$. Factorize $n$ to the form

$$
n=p^{\beta} p_{1}^{\alpha_{1}} \cdots p_{n}^{\alpha_{n}}
$$

where each $p_{i}$ is a prime and $\alpha_{i} \in \mathbb{N}$. By repeatedly using Lemma 7.4, $1 / n \in A$ if, and only if, $1 / p_{i}^{\alpha_{i}} \in A$ for all $i \in 1,2, \ldots, n$ and $1 / p^{\beta} \in A$. It follows again by repeatedly using Lemma 7.4, that

$$
\frac{1}{p_{1}^{\alpha_{1}} \cdots p_{n}^{\alpha_{n}} p^{\alpha}}=\frac{1}{n p^{\alpha-\beta}} \in A
$$

for all $\alpha \in \mathbb{N}$. Hence, as $\operatorname{gcd}(p, m)=1$ and $\operatorname{gcd}(m, n)=1$, we have

$$
\frac{m}{p_{1}^{\alpha_{1}} \cdots p_{n}^{\alpha_{n}} p^{\alpha}}=\frac{m}{n p^{\alpha-\beta}} \in A
$$

for all $\alpha \in \mathbb{N}$ with $\alpha>\beta$, and so $I_{A}(y)$ is infinite. 
Now suppose that there are infinitely many primes $q$ such that $h_{A}(q)>0$. As there are only finitely many primes dividing $m$ or $n$, it follows that there are infinitely many $k \in \mathbb{Z}$ such that $\operatorname{gcd}(k, n)=1, \operatorname{gcd}(k, m)=1$, and $k$ is a product of primes $q$ such that $h_{A}(q)>0$. Now it follows by Lemmas 7.3 and 7.4 that for all such $k$ we have $m / k n \in A$. Hence, we conclude that $I_{A}(y)$ is infinite.

Lemma 7.7 Let $G$ be an nilpotency class 2 torsion-free group with a connected power graph $P(G)$. If $H$ is an nilpotency class 2 group with $P(G) \cong P(H)$, then $\vec{P}(G) \cong$ $\vec{P}(H)$.

The proof uses the following two results; the first can be found in [2] or [7, Chapter VIII, Section 30], and the second in [8].

Proposition 7.8 Let $G$ be a group. Then the following two statements are equivalent:

- $G$ is torsion-free and locally cyclic;

- $G$ is embedded in $\mathbb{Q}$.

Proposition 7.9 Let $G$ be a torsion-free group. Then the following two statements are equivalent:

- $G$ is embedded in $\mathbb{Q}$;

- for any two non-trivial subgroups $A$ and $B$ of $G$, we have $A \cap B \neq\{1\}$.

Proof of Lemma 7.7 As $P(G) \cong P(H)$, from our previous results it follows that both $H$ and $G$ are torsion-free and locally cyclic. Hence, by Proposition 1 and Theorem 7.2 we can consider $H$ and $G$ to be unitary subgroups of $\mathbb{Q}$. Without loss of generality, we can assume $H \neq G$. By the second Proposition above, we have $G \cap H \neq\{1\}$, and since $G \cap H$ is torsion-free, it follows that $G \cap H$ contains an infinite cyclic subgroup of $\mathbb{Q}$.

Let $x \in G \cap H$. Then for all $y \in O(x)$, we have $y \in G \cap H$ (as $G \cap H$ is a subgroup of $\mathbb{Q}$ ). It follows that for $y \in H$, if there exists $x \in G \cap H$ such that $x \in I(y)$, then $y \in O(x)$, and so $y \in G \cap H$. We deduce:

For all $y \in H \backslash G$, if $x \in G \cap H$ and $x \sim y$, then $x \in O(y)$.

For such a $y$, we have $\langle y\rangle \cap(G \cap H) \neq\{1\}$ (by the second Proposition), so there exists $x \in G \cap H$ such that $x \in O(y)$. Therefore, using Lemma 3.3 we can recognize $O(y)^{\prime}$ in $N(y)^{\prime}$ as the only connected component that has an element in $G \cap H$. Hence, we can determine $I(y)^{\prime}$ as well.

Let $f$ be an isomorphism $f: P(G) \rightarrow P(H)$. Let $x \in G$ such that $x \neq 1_{G}$. If $f(x) \in H \backslash G$, then by arguments above we can determine $O(f(x))$ and $I(f(x))$ in $P(H)$. If $f(x) \in G \cap H$, then $O(f(x))=\langle f(x)\rangle \leq G \cap H$. However, we know all directions in $\vec{P}(G \cap H)$; hence, again $O(f(x))$ is determined in $P(H)$, and by looking at $N(f(x))^{\prime}$ in $P(H)^{\prime}$ and using Lemma 3.3 we can determine $I(f(x))$ as well.

By Lemma 7.6, either $I(x)$ is finite for all $x \in G$ or it is infinite for all $x \in G \backslash\{1\}$.

Consider now the first case. Let $x \in G$ be such that $x \neq 1_{G}$. Then $f(x) \neq 1_{H}$. It follows by Lemma 3.4 that for all $y \in H, I(y)$ is finite. Hence, using Lemma 3.3, as $G$ and $H$ are torsion-free, the only infinite connected components of $N(x)^{\prime}$ and $N(f(x))^{\prime}$ 
are precisely $O(x)^{\prime}$ and $O(f(x))^{\prime}$, respectively. But then again using Lemma 3.4 we deduce $f: I(x) \rightarrow I(f(x))$ and $f: O(x) \rightarrow O(f(x))$. As this is true for all $x \in G$ such that $x \neq 1_{G}$ and $f\left(1_{G}\right)=1_{H}$ we conclude that $f$ induces an isomorphism $f: \vec{P}(G) \rightarrow \vec{P}(H)$.

In the second case, we deduce by Lemma 3.4 that for all $y \in H \backslash\left\{1_{H}\right\}, I(y)$ is infinite. We find an isomorphism $f^{\prime}: \vec{P}(G) \rightarrow \vec{P}(H)$. Fix $z_{0} \in G$ such that $z_{0} \neq 1_{G}$ and let $f^{\prime}\left(z_{0}\right)=f\left(z_{0}\right)$ and $f^{\prime}\left(1_{G}\right)=1_{H}$. Let $z_{1} \in G$ such that $z_{1} \sim z_{0}$ and $z_{1} \neq z_{0}$. By the previous arguments, $O\left(f^{\prime}\left(z_{0}\right)\right)$ and $I\left(f^{\prime}\left(z_{0}\right)\right)$ are determined. Hence, if $z_{1} \in O\left(z_{0}\right)$, let $f^{\prime}\left(z_{1}\right) \in O\left(f^{\prime}\left(z_{0}\right)\right)$. Similarly, if $z_{1} \in I\left(z_{0}\right)$, let $f^{\prime}\left(z_{1}\right) \in$ $I\left(f^{\prime}\left(z_{0}\right)\right)$. Finally, if $z_{1}=z_{0}^{-1}$, let $f^{\prime}\left(z_{1}\right)=f^{\prime-1}\left(z_{0}\right)$. Then directions of the path $z^{1}=\left(z_{0}, z_{1}\right)$ agree with those of the corresponding path $f^{\prime}(z)^{1}=\left(f^{\prime}\left(z_{0}\right), f^{\prime}\left(z_{1}\right)\right)$ and $f^{\prime}:\left\{1_{G}, z_{0}, z_{1}\right\} \rightarrow H$ is an injection. We can continue in this manner to define $f^{\prime}$ in such a way that it respects the path directions. However, it remains to show that we can do so in an injective manner.

Thus, let $n \in \mathbb{N}$ and assume that the directions of the path $\left(z_{0}, z_{1}, \ldots, z_{n}\right)$ agree with those of the corresponding path $\left(f^{\prime}\left(z_{0}\right), f^{\prime}\left(z_{1}\right), \ldots, f^{\prime}\left(z_{n}\right)\right)$. Furthermore, assume that

$$
f^{\prime}:\left\{1_{G}, z_{0}, z_{1}, \ldots, z_{n}\right\} \rightarrow H
$$

is an injection. Let $z_{n+1} \in G$ be such that $z_{n+1} \sim z_{n}$ and $z_{n+1} \neq z_{n}$. Denote $S:=\left\{z_{i}: z_{i}=z_{n+1}\right\}$ and $M:=\left\{f^{\prime}\left(z_{i}\right): i \in\{1,2, \ldots, n\}\right\}$.

By our results, $O\left(f^{\prime}\left(z_{n}\right)\right)$ and $I\left(f^{\prime}\left(z_{n}\right)\right)$ are determined. If there exists $z_{i} \in S$ such that $z_{i}=z_{n+1}$, then let $f^{\prime}\left(z_{n+1}\right)=f^{\prime}\left(z_{i}\right)$, also if $z_{n+1}=z_{n}^{-1}$, let $f^{\prime}\left(z_{n+1}\right)=f^{\prime-1}\left(z_{n}\right)$. Otherwise, as $O\left(f^{\prime}\left(z_{n}\right)\right) \cup 1_{H} \cong \mathbb{Z}$ (using the fact that $f^{\prime}\left(z_{n}\right) \neq 1_{H}$ and $H$ is torsion-free), it follows that $O\left(f^{\prime}\left(z_{n}\right)\right)$ is infinite. Hence, if $z_{n+1} \in O\left(z_{n}\right)$, we can let $f^{\prime}\left(z_{n+1}\right) \in O\left(f^{\prime}\left(z_{n}\right)\right)$ such that $f^{\prime}\left(z_{n+1}\right) \notin M$. If $z_{n+1} \in I\left(z_{n}\right)$, then by our arguments above as $f^{\prime}\left(z_{n}\right) \neq 1_{H}, I\left(f^{\prime}\left(z_{n}\right)\right)$ is infinite; therefore, we can let $f^{\prime}\left(z_{n+1}\right) \in I\left(f^{\prime}\left(z_{n}\right)\right)$ such that $f^{\prime}\left(z_{n+1}\right) \notin M$. We now have that the directions of the path $\left(z_{0}, z_{1}, \ldots, z_{n+1}\right)$ agree with those of the corresponding path $\left(f^{\prime}\left(z_{0}\right), f^{\prime}\left(z_{1}\right), \ldots, f^{\prime}\left(z_{n+1}\right)\right)$. Furthermore, $f^{\prime}:\left\{1_{G}, z_{0}, z_{1}, \ldots, z_{n+1}\right\} \rightarrow H$ is an injection. Since the graph $P(G)$ is connected, we can reach any point in $P(H)$ by a path starting at $f^{\prime}\left(z_{0}\right)$. Thus, continuing in this manner we can define $f^{\prime}$ to be an isomorphism $f^{\prime}: \vec{P}(G) \rightarrow \vec{P}(H)$, as required.

Thus, we have the result (Theorem 1.3) that classifies all torsion-free nilpotency class 2 groups with respect to their power graphs:

Theorem 7.10 Let $G$ and $H$ be nilpotency class 2 torsion-free groups. Then $P(G) \cong$ $P(H)$ implies $\vec{P}(G) \cong \vec{P}(H)$.

Proof This is an immediate consequence of Lemmas 7.1 and 7.7, since an isomorphism from the power graph of $G$ to the power graph of $H$ is an isomorphism between their connected components.

It is not true in general that the power graph of a locally cyclic torsion-free group determines the group up to isomorphism. Before giving a counterexample, let us introduce new definitions. 
For a height function $h$ and a positive integer $m=p_{1}^{\alpha_{1}} \cdots p_{n}^{\alpha_{n}}$, where each $p_{i}$ is a prime and $\alpha_{i} \in \mathbb{N}$, define $m h(p)$ as the height function given by

$$
m h(p)= \begin{cases}h(p)+\alpha_{i} & \text { if } p=p_{i} \\ h(p) & \text { otherwise }\end{cases}
$$

For two height functions $h$ and $f$ we write $h \equiv f$ if, and only if, there exist nonnegative integers $m$ and $n$ such that $m h=n f$. In other words, $h \equiv f$ if and only if $h$ and $f$ differ in only finitely many positions, and they differ only finitely in these positions.

Proposition 7.11 The relation $\equiv$ is an equivalence relation.

The proof is straightforward.

Now the following is shown in [8]:

Theorem 7.12 Let $A$ and $B$ be two unitary subgroups of the rationals. Then $A \cong B$ if, and only if, $h_{A} \equiv h_{B}$.

Fix a prime $p$ and consider the subgroup of $\mathbb{Q}$, denoted by $G_{p}$, generated by all the negative powers of $p$. (It consists of all rational numbers whose denominator is a power of $p$.) Every element of $G_{p}$ can be written as a product of powers of primes, where the prime $p$ can have negative exponent but all the other exponents are non-negative. The height function of this group has the form

$$
h_{G_{p}}(q)=\left\{\begin{array}{l}
\infty \text { if } q=p \\
0 \text { otherwise. }
\end{array}\right.
$$

It follows by Theorem 7.12 that $G_{p}$ is not isomorphic to $G_{q}$ whenever $p \neq q$. However, we will show that $\vec{P}\left(G_{p}\right) \cong \vec{P}\left(G_{q}\right)$.

Theorem 7.13 Let $p$ and $q$ be two primes such that $p \neq q$. Let $\pi: P \rightarrow P$ be the transposition $\pi=(p, q)$. Then the map $\varphi: G_{p} \rightarrow G_{q}$ defined by

$$
\pm p_{1}^{\alpha_{1}} \cdots p_{n}^{\alpha_{n}} \mapsto \pm \pi\left(p_{1}\right)^{\alpha_{1}} \cdots \pi\left(p_{n}\right)^{\alpha_{n}}
$$

and $0 \mapsto 0$, induces an isomorphism $\varphi: \vec{P}\left(G_{p}\right) \rightarrow \vec{P}\left(G_{q}\right)$

Proof Observe that the map $\theta: G_{q} \rightarrow G_{p}$ given by

$$
\pm p_{1}^{\alpha_{1}} \cdots p_{n}^{\alpha_{n}} \mapsto \pm \pi^{-1}\left(p_{1}\right)^{\alpha_{1}} \cdots \pi^{-1}\left(p_{n}\right)^{\alpha_{n}}
$$

and $0 \mapsto 0$, is an inverse of $\varphi$. Hence, $\varphi$ is a bijection. Let $a, b \in G$ be such that $a \rightarrow b$ in $\vec{P}\left(G_{p}\right)$. Factorize $a$ and $b$ as

$$
\begin{aligned}
& a= \pm 2^{\alpha_{1}} 3^{\alpha_{2}} \cdots p_{i}^{\alpha_{i}} \cdots, \\
& b= \pm 2^{\beta_{1}} 3^{\beta_{2}} \cdots p_{i}^{\beta_{i}} \cdots,
\end{aligned}
$$


where each $p_{i}$ is a prime and the exponents are allowed to be zero. By our assumption, there exists $m \in \mathbb{Z}$ such that $b=m a$. However, this is equivalent to saying that $\alpha_{i} \leq \beta_{i}$ for all $i$. Now as

$$
\begin{aligned}
& \varphi(a)= \pm \pi(2)^{\alpha_{1}} \pi(3)^{\alpha_{2}} \cdots \pi\left(p_{i}\right)^{\alpha_{i}} \cdots, \\
& \varphi(b)= \pm \pi(2)^{\beta_{1}} \pi(3)^{\beta_{2}} \cdots \pi\left(p_{i}\right)^{\beta_{i}} \cdots,
\end{aligned}
$$

and $\alpha_{i} \leq \beta_{i}$ for all $i$, it follows that $\varphi(a) \rightarrow \varphi(b)$ in $\vec{P}\left(G_{q}\right)$. This is true for all such $a, b \in G_{p}$ and $\varphi(0)=0$. Similarly, $a \rightarrow b$ if $\varphi(a) \rightarrow \varphi(b)$. It follows that $\varphi$ induces the required isomorphism.

\section{Open problem}

We mention a problem which we have been unable to solve.

If $G$ is a torsion-free nilpotent group of class 2 , and $H$ a group with $P(G) \cong P(H)$, is it true that $\vec{P}(G) \cong \vec{P}(H)$ ?

\section{References}

1. Aalipour, G., Akbari, S., Cameron, P.J., Nikandish, R., Shaveisi, F.: On the structure of the power graph and the enhanced power graph of a group. https://arxiv.org/abs/1603.04337

2. Baer, R.: Abelian groups without elements of finite order. Duke Math J. 3, 68-122 (1937)

3. Cameron, P.J.: The power graph of a finite group, II. J. Group Theory 13, 779-783 (2010)

4. Cameron, P.J., Ghosh, S.: The power graph of a finite group. Discrete Math. 311, 1220-1222 (2011)

5. Chakrabarty, Ivy, Ghosh, Shamik, Sen, M.K.: Undirected power graphs of semigroups. Semigroup Forum 78, 410-426 (2009)

6. Kelarev, A.V., Quinn, S.J.: Directed graph and combinatorial properties of semigroups. J. Algebra 251, 16-26 (2002)

7. Kurosh, A.: Group Theory, 2nd English edn (transl. K.A Hirsch). Chelsea, New York (1960)

8. Miller, P.: A classification of the subgroups of the rationals under addition. https://www.whitman.edu/ Documents/Academics/Mathematics/SeniorProject_PatrickMiller.pdf

9. Neumann, B.H.: An essay on free products with amalgamations. Philos. Trans. R. Soc. Lond Ser. A. 246, 503-544 (1954) 